\title{
Machine learning-based random forest predicts anastomotic leakage after anterior resection for rectal cancer
}

\author{
Rongbo Wen ${ }^{1 \#}$, Kuo Zheng ${ }^{1 \#}$, Qihang Zhang ${ }^{2 \#}$, Leqi Zhou ${ }^{1 \#}$, Qizhi Liu ${ }^{1}$, Guanyu Yu $^{1}$, Xianhua Gao ${ }^{1}$, \\ Liqiang Hao ${ }^{1}$, Zheng Lou ${ }^{1}$, Wei Zhang ${ }^{1}$ \\ ${ }^{1}$ Department of Colorectal Surgery, Changhai Hospital, Shanghai, China; ${ }^{2}$ School of Industrial and Systems Engineering, Georgia Institute of \\ Technology, Atlanta, Georgia, USA \\ Contributions: (I) Conception and design: Wei Zhang, Zheng Lou; (II) Administrative support: X Gao, L Hao; (III) Provision of study materials \\ or patients: G Yu, Q Liu; (IV) Collection and assembly of data: K Zheng, L Zhou; (V) Data analysis and interpretation: Q Zhang, R Wen; \\ (VI) Manuscript writing: All authors; (VII) Final approval of manuscript: All authors. \\ \#These authors contributed equally to this work. \\ Correspondence to: Wei Zhang, MD, PhD; Zheng Lou, MD, PhD. Department of Colorectal Surgery, Changhai Hospital, 168 Changhai Rd., \\ Shanghai 200433, China. Email: weizhang2000cn@163.com; louzhengpro@126.com.
}

Background: Anastomotic leakage (AL) is one of the commonest and most serious complications after rectal cancer surgery. The previous analyses on predictors for AL included small-scale patients, and their prediction models performed unsatisfactorily.

Methods: Clinical data of 5,220 patients who underwent anterior resection for rectal cancer were scrutinized to create a prediction model via random forest classifier. Additionally, data of 836 patients served as the test dataset. Patients diagnosed with AL within 6 months' follow-up were recorded. A total of 20 candidate factors were included. Receiver operating characteristic (ROC) curve was conducted to determine the clinical efficacy of our model, and compare the predictive performance of different models.

Results: The incidence of AL was 6.2\% (326/5,220). A multivariate logistic regression analysis and the random forest classifier indicated that sex, distance of tumor from the anal verge, bowel stenosis or obstruction, preoperative hemoglobin, surgeon volume, diabetes, neoadjuvant chemoradiotherapy, and surgical approach were significantly associated with AL. After propensity score matching, the temporary stoma was not identified as a protective factor for $\mathrm{AL}(\mathrm{P}=0.58)$. Contrastingly, the first year of performing laparoscopic surgery was a predictor $(\mathrm{P}=0.009)$. We created a predictive random forest classifier based on the above predictors that demonstrated satisfactory prediction efficacy. The area under the curve (AUC) showed that the random forest had higher efficiency (AUC =0.87) than the nomogram (AUC =0.724).

Conclusions: Our findings suggest that eight factors may affect the incidence of AL. Our random forest classifier is an innovative and practical model to effectively predict AL, and could provide rational advice on whether to perform a temporary stoma, which might reduce the rate of stoma and avoid the ensuing complications.

Keywords: Anastomotic leakage (AL); rectal cancer; risk factor; machine learning; nomogram; random forest

Submitted Oct 10, 2020. Accepted for publication Mar 28, 2021.

doi: 10.21037/jgo-20-436

View this article at: http://dx.doi.org/10.21037/jgo-20-436

\section{Introduction}

Colorectal cancer (CRC) is the third leading cause of cancer-related deaths according to a latest statistical report (1). Although improvements have been successful at reducing the incidence of CRC (2), the morbidity and mortality still remain high. Anterior resection (AR) based on total mesorectal excision (TME) (3) is the major surgical treatment for rectal cancer. 
Anastomotic leakage (AL) is one of the most common and serious complications after AR for rectal cancer. The incidence of AL varies from $3 \%$ to $21 \%$ according to previous reports (4-7). Patients with AL are likely to suffer from increased length of hospital stay and rates of re-operation, and even recurrence and mortality. Some of the patients may even require a de-functioning stoma that would significantly affect their quality of life. A temporary stoma is often recommended for patients with high risk of AL (8). However, the necessity of a temporary stoma is hard to decide upon since AL is associated with many factors, and is difficult to be predicted.

Though multiple studies have reported the predictors for $\mathrm{AL}(5,6,9)$, accurate prediction of AL remains difficult. According to the current knowledge, the healing of the anastomotic site depends on the tension and blood supply around the anastomotic site (10). The previous reports of risk factors included relatively few patients, and their results were not consistent (5,7,11-13). Moreover, the performance of the established models predicting the incidence of AL remains unsatisfactory. Random forest, a new and highly flexible machine learning algorithm, has wide application prospects, and has been demonstrated to have better performance in disease prediction (14). However, to the best of our knowledge, no article has reported the application of random forest in AL prediction so far. In this study, we aimed to analyze a large number of rectal cancer patients after AR to illustrate the risk factors of AL, and to create a random forest classifier to better predict the incidence of AL and give an advice on whether to a do temporary stoma. We present the following article in accordance with the TRIPOD reporting checklist (available at http://dx.doi. org/10.21037/jgo-20-436).

\section{Methods}

The study was conducted in accordance with the Declaration of Helsinki (as revised in 2013). The study was approved and monitored by the Ethics Committee of Changhai Hospital (No. CHEC2020-035). Because of the retrospective nature of the study, the requirement for informed consent was waived.

\section{Patients}

Patients who underwent AR for rectal cancer in Shanghai Changhai Hospital from August 2009 to June 2018, were included in the study. In addition, data of patients who underwent AR from July 2018 to June 2019, in our institution were collected as a group for external validation. Demographics, clinicopathological variables, and followup were extracted from the prospectively maintained CRC database.

\section{Inclusion and exclusion criteria}

Enrolled patients met the following inclusion criteria: rectal cancer patients; underwent AR with TME; complete clinical data. The exclusion criteria included: patients who underwent local excision, Miles or Hartmann surgery; patients with tumor $>15 \mathrm{~cm}$ from the anal verge; patients with multiple primary colorectal carcinomas.

\section{Diagnosis of $A L$}

AL was defined as the defect of the intestinal wall at the anastomotic site (including suture and staple lines of neorectal reservoirs) leading to a communication between the intra- and extra-luminal compartments (15). A pelvic abscess close to the anastomosis was also considered as AL. Based on its impact on clinical management, AL was classified into three grades (grade A, B, and C) according to the International Study Group of Rectal Cancer: Grade A resulted in no change in patients' management, whereas grade B leakage required active clinical intervention but was manageable without re-operation. Grade $\mathrm{C}$ required re-operation (15). In this study, AL was diagnosed via digital rectal examination (DRE), endoscopy, or imaging examination within 6 months. The follow-up was conducted via outpatient or telephone.

\section{Variables}

Demographic variables were defined and analyzed as follows: sex, age at operation, body mass index (BMI). Clinicopathological variables were diabetes, preoperative albumin (pALB) ( $<35 v s . \geq 35 \mathrm{~g} / \mathrm{L})$, preoperative hemoglobin (pHGB) (<90 vs. $\geq 90 \mathrm{~g} / \mathrm{L})$, carcinoembryonic antigen (CEA) ( $>5 v s . \leq 5 \mathrm{ng} / \mathrm{mL}$ ), carbohydrate antigen 199 (CA199) (>37 $v s . \leq 37 \mathrm{U} / \mathrm{mL}$ ), preoperative bowel stenosis or obstruction, surgical approach (laparoscopic or open), blood loss, blood transfusion, distal tumor distance from the anal verge, neoadjuvant chemoradiotherapy (nCRT), surgeon volume (high volume surgeon: amount of colorectal surgeries $>100$ in the previous year; a total of 8 well-trained surgeons were include, qualifications of surgeons can be seen in Table S1), 


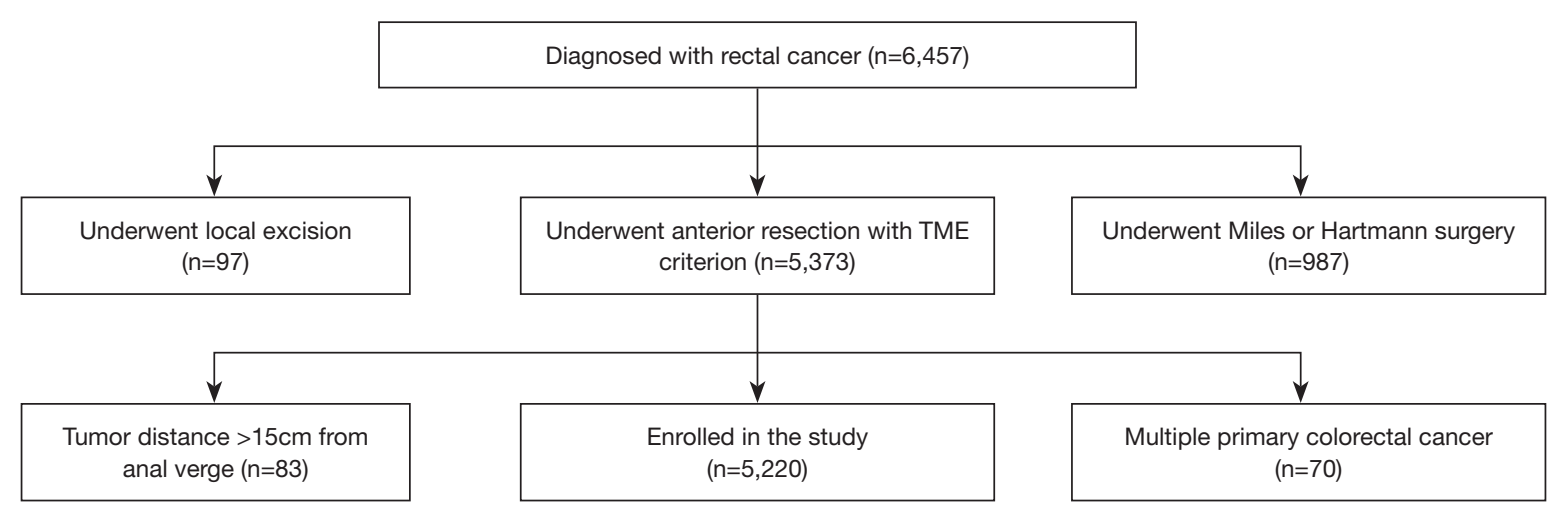

Figure 1 The flow chart of the selection process of patients.

temporary stoma, pathological $\mathrm{T}$ stage, pathological $\mathrm{N}$ stage, pathological types, and American Society of Anesthesiologists (ASA) score. The above variables were selected as candidate predictors because of previous reports and clinical experiences.

\section{Statistical analysis}

Statistical analyses were conducted with the statistical package for social sciences (SPSS version 22.0.0, IBM SPSS statistics, IBM Corporation, Armonk, NY, USA), R software (version 3.5.1; http://www.Rproject.org), and Python software (version 3.7.4; https://www.python.org). Descriptive statistics were computed for all variables. These included means and standard deviations (SD) for continuous factors, and frequencies for categorical factors. Comparisons of the distribution of clinicopathological characteristics were performed by using the two-tailed $t$-test (or Wilcoxon rank sum test as appropriate) for continuous variables and chi-square test (or the Fisher exact test as appropriate) for categorical variables. To evaluate whether temporary stoma was a risk factor for AL, propensity score matching (PSM) was implemented to reduce the possibility of selection bias by using a logistic regression model (16). P values of 0.05 or lower were considered statistically significant.

Candidate predictors incorporated in the prediction nomogram were based on the multivariate logistic regression analysis. In this study, the random forest classifier was also applied for which all the categorical data were transformed into numerical values in order to train the model. The pre-processed data set was then split into training set and validation set. Grid-search cross validation technique was used to tune the number of estimators in the classifier, and all trainings were conducted with 5 -fold cross validation to prevent overfitting.
Receiver operating characteristic (ROC) curve was conducted to evaluate the clinical usefulness of the nomogram and the random forest classifier by comparing the area under the curve (AUC) formed by the real results and the predicted results (17).

\section{Results}

\section{Demographic and clinicopathological characteristics of the patients}

A total of 5,220 eligible patients were enrolled (Figure 1), including $3,454(66.2 \%)$ patients with a temporary stoma. 1,824 patients $(34.9 \%)$ were female and 3,396 patients (65.1\%) were male. Patients with AL classified as Grade B accounted for $3.83 \%(200 / 5,220)$, while those classified as grade A and grade C accounted for $1.86 \%(97 / 5,220)$ and $0.56 \%(29 / 5,220)$, respectively. Additionally, 1,343 patients $(25.7 \%)$ received nCRT and 896 patients $(17.2 \%)$ underwent laparoscopic surgery. The overall incidence of AL was $6.25 \%(326 / 5,220)$ (Table 1$)$.

In the test dataset with 836 patients, the incidence of clinical AL was $5.4 \%$ (45/836). Additionally, $26.6 \%$ of these patients received nCRT (222/836), which was higher than that in the training dataset. Further, 22.7\% (190/836) of these patients underwent laparoscopic surgery, which was also higher than that in the training dataset. Similarly, the proportion of patients with temporary stoma in the test dataset was also higher than that in the training dataset (69.2\% vs. 66.2\%, respectively). Conversely, the distance of tumor from the anal verge was lower in the test set $(6.53 \pm 3.28 v s$. $6.99 \pm 3.18$, respectively). The reasons for the differences regarding temporary stoma and nCRT between the two datasets were owing to the development 
Table 1 Univariate analysis of variables related to anastomotic leakage $(\mathrm{n}=5,220)$

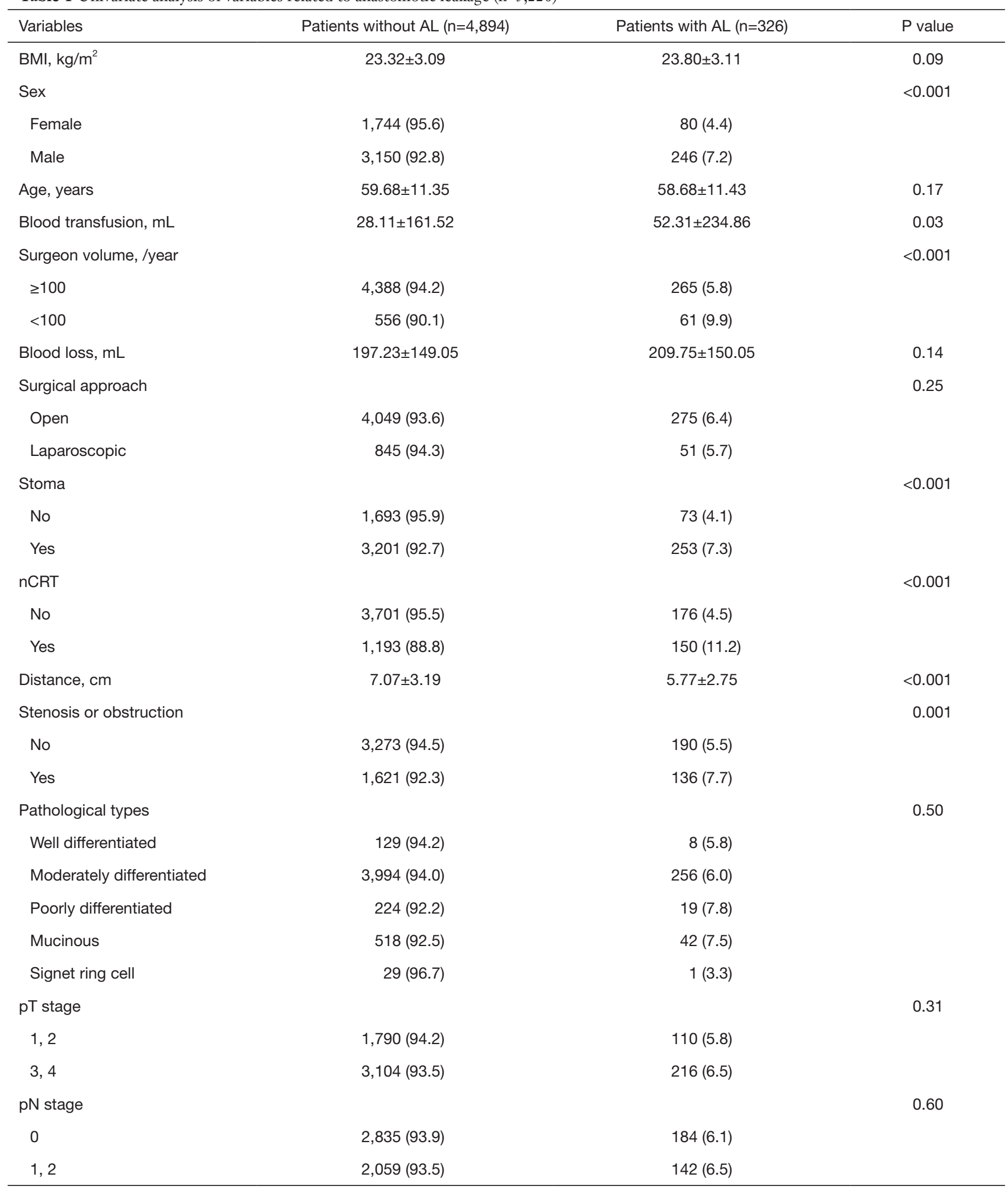

Table 1 (continued) 
Table 1 (continued)

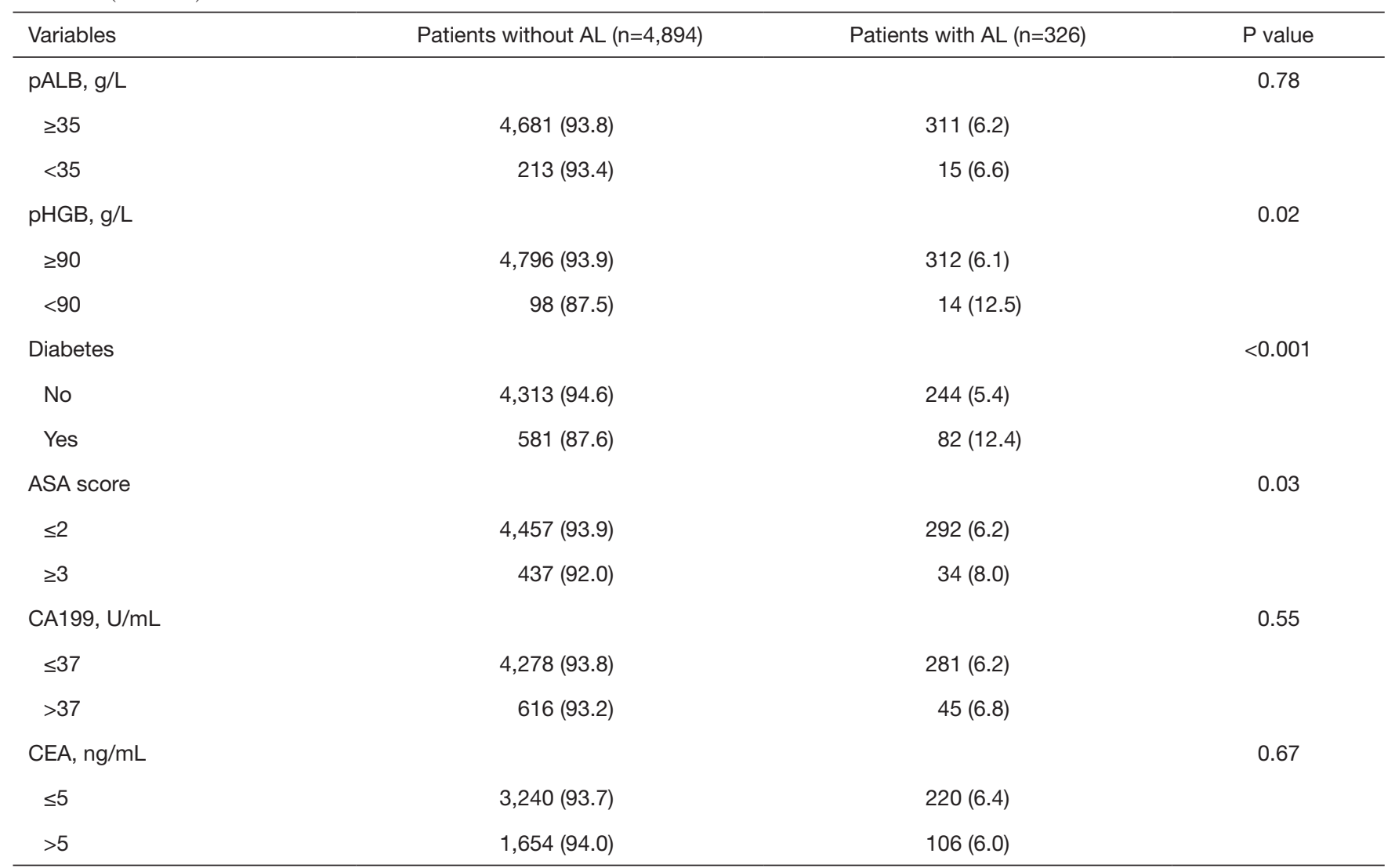

Data were presented as $\mathrm{n}(\%)$ or mean \pm standard deviation. AL, anastomotic leakage; BMI, body mass index; nCRT, neoadjuvant chemoradiotherapy; pALB, preoperative albumin; pHGB, preoperative hemoglobin; ASA, American Society of Anesthesiologists; CEA, carcinoembryonic antigen; CA199, carbohydrate antigen 199.

of the concepts of nCRT, and techniques in laparoscopic surgery during the past few years. Patients who received neoadjuvant therapy or underwent low AR were more likely to receive a temporary stoma during surgery (Table S2).

\section{Risk factors associated with $A L$}

According to univariate analysis, of all the examined variables, sex, blood transfusion volume, temporary stoma, surgeon volume, nCRT, distance of tumor from the anal verge, bowel stenosis or obstruction, pHGB level, diabetes, and ASA score were associated with AL (Table 1). Moreover, we found that the incidence of $\mathrm{AL}$ was significantly higher during the surgeon's first year of laparoscopic surgery compared with the following years $(\mathrm{P}=0.009)$ (Table 2). Therefore, the surgical approach was re-defined as open surgery, first year, and following years of laparoscopic surgery. The risk factors with $\mathrm{P}<0.2$ in the univariate analysis were included in the multivariate analysis. The multivariate analysis showed that sex, surgeon volume, distance of tumor from the anal verge, bowel stenosis or obstruction, pHGB, nCRT, diabetes, and surgical approach were independent risk factors of $\mathrm{AL}$ (Table 3).

As shown in Table 1, patients with temporary stoma appeared to have a higher incidence of AL, which was contradictory to a previous report (18). To further confirm whether temporary stoma is associated with AL, we used PSM to balance the baseline data. According to the univariate analysis, BMI, sex, blood transfusion, blood loss, distance of tumor from the anal verge, nCRT, surgical approach, and pALB were considered as the matching variables (Table S3). A total of 2,218 patients were selected after PSM (ratio $=1: 1$, caliper $=0.05$ ), and there was no significant difference between patients with and without temporary stoma regarding the incidence of $\mathrm{AL}(\mathrm{P}=0.58)$ (Table 4). 
Table 2 Relationship between laparoscopic year of surgeon and AL

\begin{tabular}{|c|c|c|c|}
\hline Laparoscopic year of surgeon & Patient without $\mathrm{AL}$ & Patient with $\mathrm{AL}$ & $P$ value \\
\hline 1 & $74(88.1 \%)$ & $10(11.9 \%)$ & \\
\hline 2 & $151(94.4 \%)$ & $9(5.6 \%)$ & \\
\hline 3 & $195(97.0 \%)$ & $6(3.0 \%)$ & \\
\hline 5 & 139 (93.9\%) & $9(6.1 \%)$ & \\
\hline 6 & 95 (97.9\%) & $2(2.1 \%)$ & \\
\hline 7 & $62(93.9 \%)$ & $4(6.1 \%)$ & \\
\hline
\end{tabular}

$\mathrm{AL}$, anastomotic leakage.

Table 3 Independent risk factors of AL according to multivariate analysis

\begin{tabular}{lcc}
\hline Variables & OR $(95 \% \mathrm{Cl})$ & P value \\
\hline Sex & $1.792(1.369-2.345)$ & $<0.001$ \\
Surgeon volume & $2.757(2.000-3.800)$ & $<0.001$ \\
nCRT & $3.498(2.718-4.501)$ & $<0.001$ \\
Distance of tumor to anal verge & $0.851(0.815-0.890)$ & $<0.001$ \\
Stenosis or obstruction & $1.572(1.235-2.001)$ & $<0.001$ \\
pHGB level & $2.949(1.579-5.508)$ & 0.001 \\
Diabetes & $3.385(2.543-4.506)$ & $<0.001$ \\
Surgical approach & - & $<0.001$ \\
\hline
\end{tabular}

$\mathrm{AL}$, anastomotic leakage; OR, odds ratio; $\mathrm{Cl}$, confidence interval; $\mathrm{nCRT}$, neoadjuvant chemoradiotherapy; pHGB, preoperative hemoglobin.

Table 4 Relationship between stoma and AL after PSM $(n=2,366)$

\begin{tabular}{lccc}
\hline Stoma & Patient without AL & Patient with AL & P value \\
\hline No & $1,125(95.1 \%)$ & $58(4.9 \%)$ & \\
Yes & $1,118(94.5 \%)$ & $65(5.5 \%)$ & 0.58 \\
\hline
\end{tabular}

$\mathrm{AL}$, anastomotic leakage; PSM, propensity score matching.

\section{Prediction model for $A L$}

A random forest classifier that is a classic machine learning approach was constructed to predict the incidence of AL. All the risk factors with $\mathrm{P}<0.2$ in univariate analysis were included in the random forest model, and their respective feature importance in the model were calculated. Since the number of positive class (AL patients) was much less than that of the negative class (that we only have 326 positive labels out of 5,220 patients), we up-sampled patients from positive class with replacement to solve the imbalanced class problem. After resampling, we obtained a new dataset containing 9,788 patients' information with the same numbers of positive and negative classes.

The top 8 factors with the greatest impact weights were chosen into the final random forest model (Table 5). In order to prevent overfitting, we performed 5 -fold crossvalidation to train the model. During each training, $80 \%$ of the patients were sampled to form the training set, and the rest $20 \%$ patients were left as the validation set.

To evaluate our model, we used AUC-ROC curve, specificity, and sensitivity as our evaluation metrics. The average AUC for the training set was $0.89 \pm 0.00$ (Figure $2 A$ ) while that for the validation set was $0.85 \pm 0.02$ (Figure $2 B$ ). The sensitivity of training and validation set was 0.827 and 0.818 , respectively, and the specificity was 0.739 and 0.67 , 
Table 5 Weight of factors in random forest

\begin{tabular}{lc}
\hline Factor & Weight \\
\hline Distance of tumor to anal verge & 0.316318 \\
$\mathrm{nCRT}$ & 0.286645 \\
Diabetes & 0.173374 \\
Surgeon volume & 0.081505 \\
Sex & 0.070168 \\
Stenosis or obstruction & 0.027725 \\
Preoperative hemoglobin & 0.018607 \\
Surgical approach & 0.017111 \\
\hline
\end{tabular}

nCRT, neoadjuvant chemoradiotherapy.

A
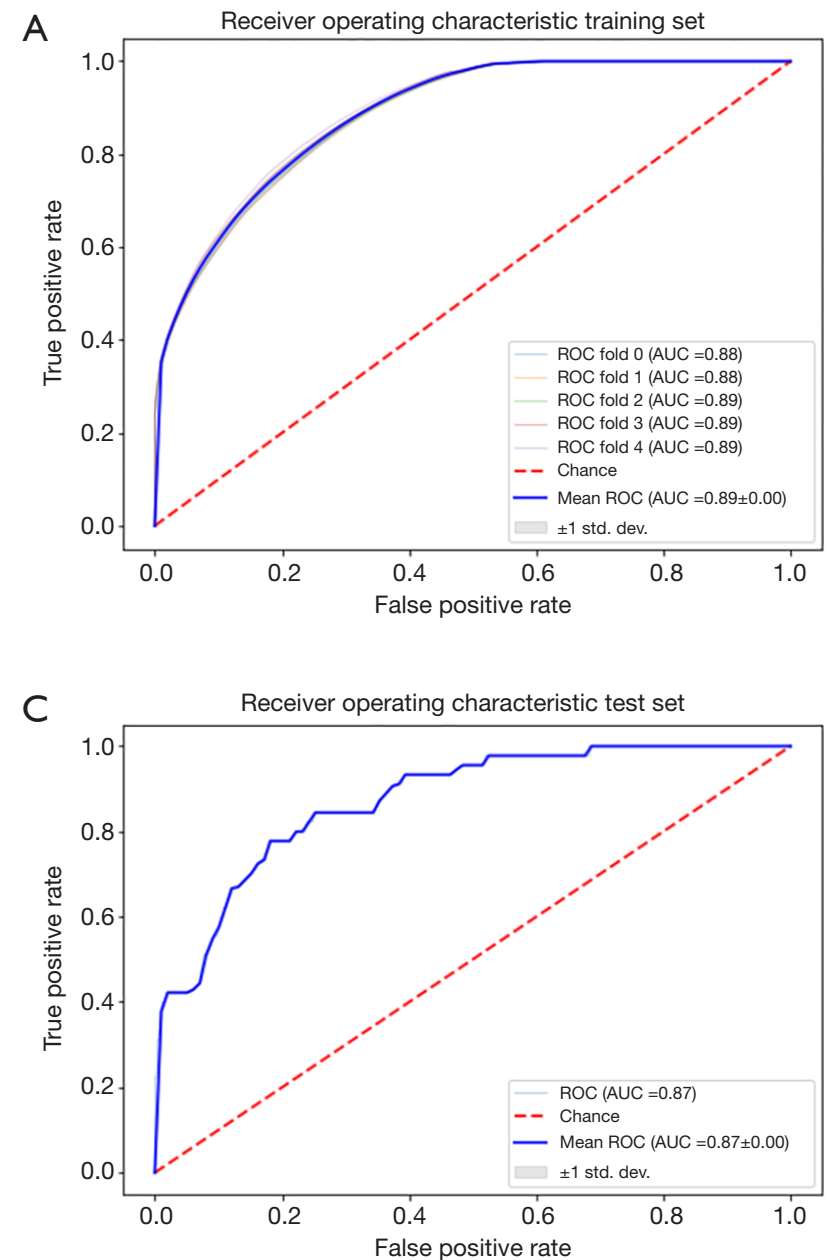

respectively. According to the data in the test set, the AUC was 0.87 , while the sensitivity and specificity were 0.844 and 0.697, respectively (Figure 2C).

In order to verify the efficacy of the random forest classifier, we also constructed a nomogram to predict AL based on the same clinical data (Figure 3). Patients from August, 2009, to June, 2018, were collected as training set while patients from July, 2018, to June, 2019, were regarded as test set. Based on the multivariate analysis, sex, surgeon volume, distance of tumor from the anal verge, bowel stenosis or obstruction, pHGB level, nCRT, diabetes, and surgical approach were included in the final nomogram. The AUC of training and test set was 0.735 and 0.724 ,

B

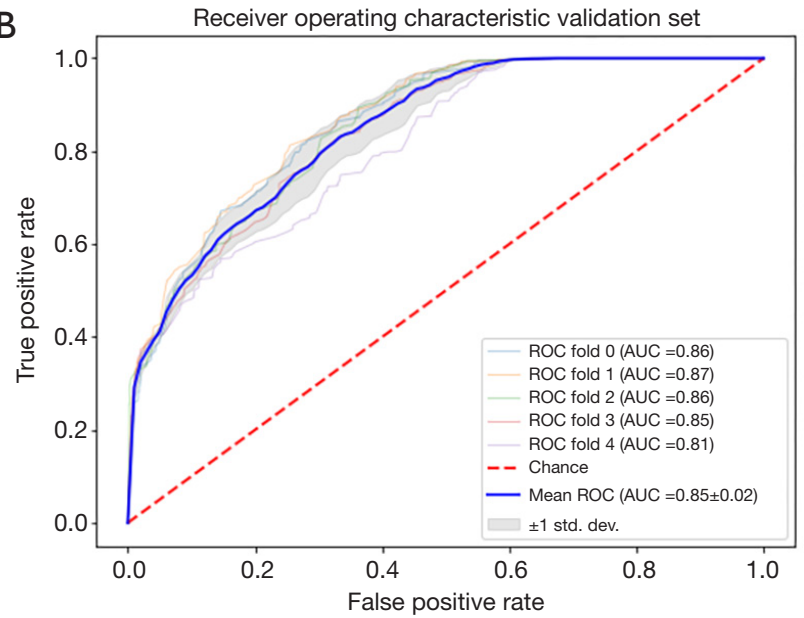

Figure 2 The ROC curve was to validate the efficiency of Random forest model. (A) Eighty percent patients ( $\mathrm{n}=4,176)$ were split into training set in each fold. Five-fold cross-validation was applied in the random forest model. Each iteration produced an ROC curve. The average AUC for the training set was $0.89 \pm 0.00$; (B) $20 \%$ patients $(n=1,044)$ were split into validation set in each fold. The average AUC for the validation set was $0.85 \pm 0.02$; (C) 836 patients were prospectively collected as a group for external validation. The AUC for the test set was 0.87 . ROC, receiver operating characteristic; AUC, area under the curve. 


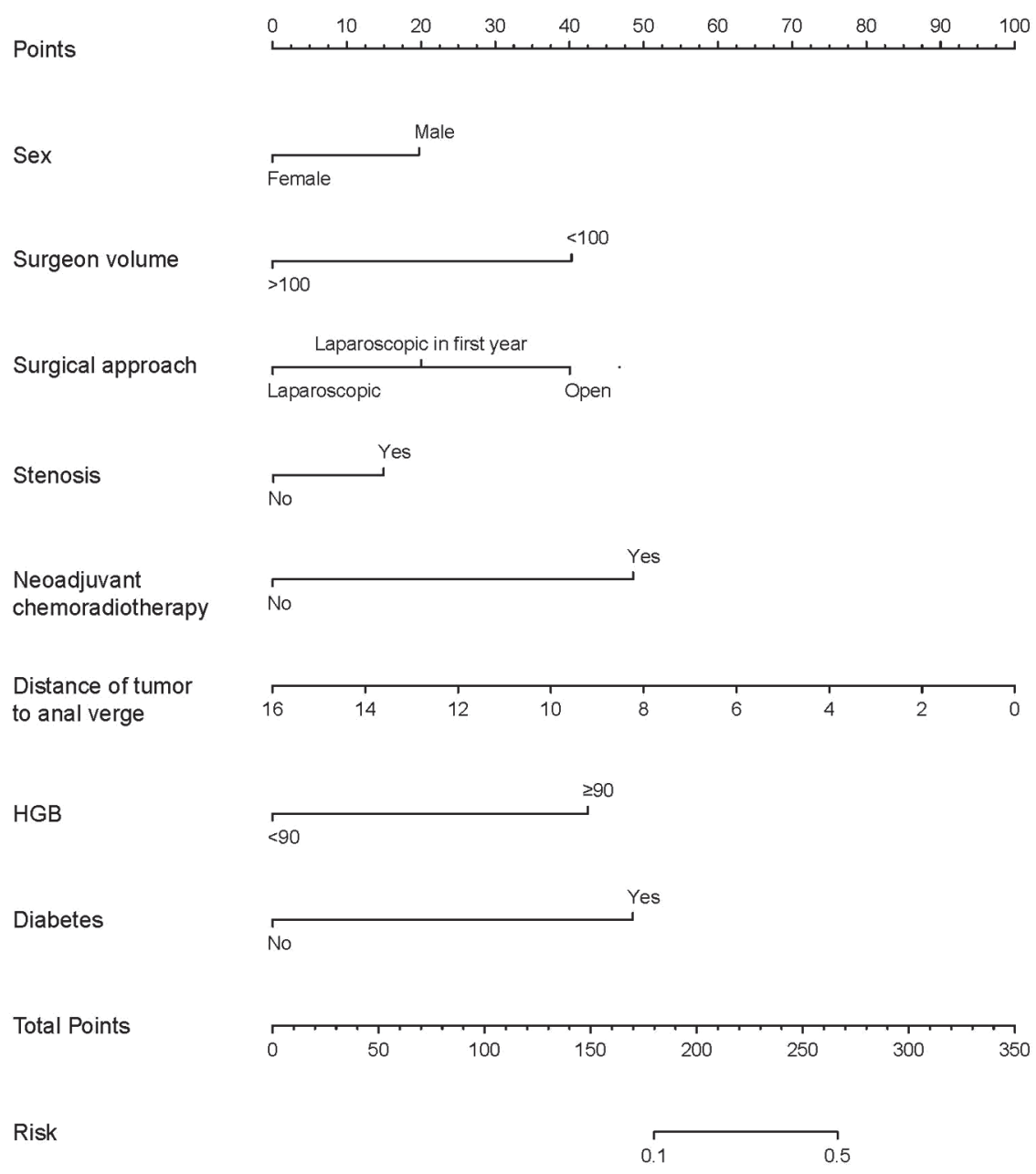

Figure 3 Nomogram to calculate risk scores and predict AL probability for RC patients. To estimate the probability of AL in a given patient, mark patient values at each axis, draw a straight line perpendicular to the point axis, and sum the points for all variables. Then, we summed the total points and drew vertical line from the total points row to obtain the probability of AL. AL, anastomotic leakage; RC, rectal cancer.

respectively, which was inferior to that of the random forest classifier (Figure 4A,B).

The final random forest classifier has been stored and can be used for future applications. Moreover, we are constructing a website embedded with the model and user instructions so that future doctors can further validate and utilize our model to predict AL with our model as the reference (http://www.changhai-rc-al-prediction.org).

\section{Discussion}

In this study, 5,220 patients who underwent AR for rectal cancer were enrolled and analyzed to identify the predictors for AL. We collected 20 demographic and clinicopathological characteristics to identify the predictors for AL. After univariate and multivariate analysis, 8 predictors for AL were identified as follows: sex, surgeon volume, distance of tumor from the anal verge, bowel stenosis or obstruction, pHGB, nCRT, diabetes, and surgical approach. Furthermore, we created a random forest classifier, which is a part of machine learning, providing a more accurate prediction for the risk of $\mathrm{AL}$ ( $\mathrm{AUC}=0.87$ ) than the nomogram $(\mathrm{AUC}=0.724$ ), which is based on logistic regression model. To the best of our knowledge, our study is the largest retrospective singlecenter study according to the data volume so far.

In our study, AUC and ROC curves confirmed that the random forest model, based on predictors per our findings, had greater predictive efficiency than the nomogram, which 

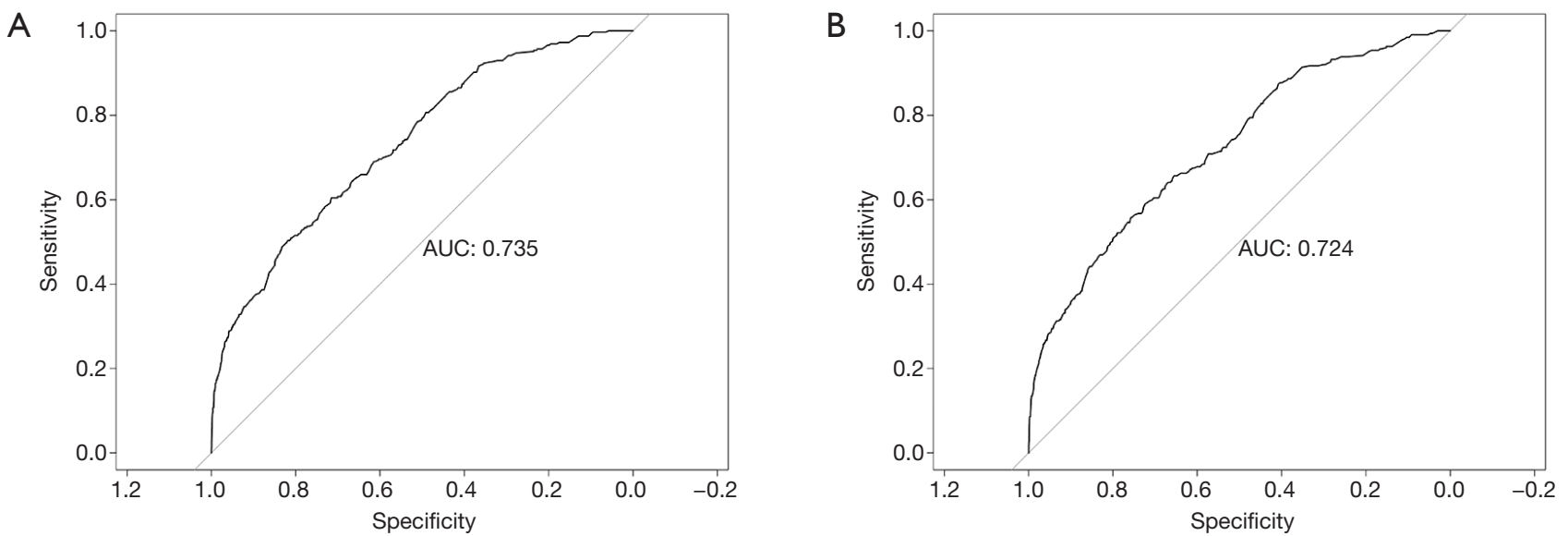

Figure 4 The ROC curve was applied to validate the efficiency of the prediction nomogram. (A) The AUC was 0.735 (n=5,220) in the training set; (B) the AUC was $0.724(\mathrm{n}=836)$ in the test set.

Table 6 Prediction performance of different models

\begin{tabular}{|c|c|c|c|c|c|}
\hline Author & Number of patients & Number of risk factors & AUC or C-index & Sensitivity & Specificity \\
\hline Nobuaki Hoshino et al. & 936 & 5 & 0.72 & - & - \\
\hline Johannes Klose et al. & 972 & 6 & 0.686 & - & - \\
\hline Chanchan Xiao et al. & 477 & 5 & 0.816 & $66.67 \%$ & $86.09 \%$ \\
\hline Seung Up Yang et al. & 566 & 5 & 0.803 & $95.7 \%$ & $67 \%$ \\
\hline Tenghui Ma et al. & 321 & 3 & 0.722 & - & - \\
\hline Our model & 5,220 & 8 & 0.850 & $81.8 \%$ & $67 \%$ \\
\hline
\end{tabular}

was widely used in the previous studies $(5,6,11,12,19)$. However, the principle of the nomogram to predict $\mathrm{AL}$ is based on logistic regression that has limitations in the fitting of model creation (20). Nevertheless, machine learning that derives from the computer field has been widely used in different fields, and can partly overcome the limitations of the regression models $(21,22)$. Random forest is a type of machine learning based on decision tree algorithm, and it has shown better predictive value than the traditional prediction model (23). However, to the best of our knowledge, no prediction model of random forest for $\mathrm{AL}$ has been reported to date. We constructed the random forest model to predict the incidence of AL for the first time and with the largest data volume $(n=5,220)$. To prevent our model from overfitting, we performed cross-validation to generate robust results (24). Thus, the results in our study are likely to be more reliable and convincing. Moreover, compared to other studies, our model also showed a better predicting performance $(11,12,19,20,25-27)$ (Table 6).

Of all enrolled patients who underwent AR for rectal cancer in this study $(\mathrm{n}=5,220), 326(6.2 \%)$ patients were diagnosed with AL. In previous studies, there were significant variations in the incidence of AL after rectal resection, ranging from $3 \%$ to $21 \%(5-7)$. $\mathrm{AL}$ is more likely to occur in the left colon and rectal surgeries than in the right colon surgery (9). Clinical AL gains more attention not only because it is easy to be detected but also for its impact on follow-up treatment.

Whether a temporary stoma can reduce the incidence of AL is debatable (18). Some studies believed that a temporary stoma could indirectly accelerate anastomotic healing, thus reducing the incidence of AL (4). Other 
studies discovered that a temporary stoma could not reduce the incidence of AL, but could relieve the difficulty in managing postoperative complications (7). In this study, we used PSM to balance the differences of baseline data between the stoma and non-stoma groups. After PSM, we found that there was no correlation between stoma and AL, which indicated that a temporary stoma could not decrease the incidence of AL. However, our data showed that $37.0 \%$ (27/73) patients with AL needed re-operation in the nonstoma group, while in the stoma group, the re-operation rate was $0.8 \%(2 / 253)$ (Table S4). It was a hint for surgeons that a temporary stoma could reduce the incidence of reoperation necessitated by AL. Besides, the stoma rate was $66.2 \%$ in our study which was higher than several other studies $(12,20,25)$. In contrast, in our study the average distance of tumor from the anal verge was lower than that in most previous studies (around $7.5 \mathrm{~cm})(12,20,25,26)$. In our study, the overall average distance was $6.99 \pm 3.18 \mathrm{~cm}$, and that in the stoma group was $5.75 \pm 2.52 \mathrm{~cm}$, while in non-stoma group that was $9.41 \pm 2.90 \mathrm{~cm}$, which implied that there were more low rectal cancer patients in our study.

Distance of tumor from the anal verge and sex are widely acknowledged as risk factors for AL in previous studies (28). Consistently, our study showed that male patients and those with lower distance of tumor from the anal verge were more likely to have AL. Similarly, preoperative bowel stenosis or obstruction, preoperative anemia $(<90 \mathrm{~g} / \mathrm{L})$ or massive blood loss during operation, diabetes, and nCRT that were also considered as predictors in our study, have also been reported in several other studies $(19,29)$. A proximal bowel stenosis or even obstruction may induce the proximal and distal bowel tissue edema, and thus increase the incidence of AL after AR. Patients with anemia or diabetes may have reduced blood supply, and tend to have a high risk of infection that may affect wound healing negatively (5). The nCRT may damage the tissue of gut lumen, and even impair the sphincter's functioning, thus delaying the anastomotic healing. In addition to the above predictive factors, we also found that surgeon volume, and surgical approach was related to the incidence of AL. It was argued that low volume surgeons were more likely to incur $\operatorname{AL}(29,30)$, and we found the same conclusion. However, when it comes to surgical approach, except open surgery $(12,29)$, performing laparoscopic surgery in first year could also raise the incidence of AL. This result indicates that we cannot neglect the human factor in high volume surgeons and laparoscopic learning curve may contribute to AL (30-32).

However, there were also some limitations in our study.
This was a retrospective study in a single center, which might have caused selection bias for patients and lacked external validation of other centers. We are now conducting prospective multicenter study to further verify our model through website. Besides, other relevant variables, such as smoking, were not recorded in the database. Whether the status of KRAS is associated with AL is controversial (13). However, because of the missing data of KRAS in early years, we only collected 3,806 patients with the accurate status of KRAS and found that there was no correlation between the status of KARS and AL ( $\mathrm{P}=0.784)$ (Table S5).

\section{Conclusions}

Our study suggests that eight factors are closely related to the incidence of AL. Our prediction model of random forest may be a practical tool for more accurate prediction of AL after AR for rectal cancer. Machine learning combined with big data has great application prospects in predicting AL. The results of our random forest model could also provide a rational advice on whether to do a temporary stoma, which might reduce the high rate of stoma and avoid the ensuing complications.

\section{Acknowledgments}

Funding: This study was supported by Clinical Science and Technology Innovation Project of Shanghai Shenkang Hospital Development Center (SHDC12016122), 234 Climbing the Discipline Program of first affiliated hospital of Naval Medical University (2019YXK032) and Ethicon Excellence in Surgery Grant (HZB-20181119-30).

\section{Footnote}

Reporting Checklist: The authors have completed the TRIPOD reporting checklist. Available at http://dx.doi. org/10.21037/jgo-20-436

Data Sharing Statement: Available at http://dx.doi. org/10.21037/jgo-20-436

Peer Review File: Available at http://dx.doi.org/10.21037/ jgo-20-436

Conflicts of Interest: All authors have completed the ICMJE uniform disclosure form (available at http://dx.doi. org/10.21037/jgo-20-436). The authors have no conflicts of 
interest to declare.

Ethical Statement: The authors are accountable for all aspects of the work in ensuring that questions related to the accuracy or integrity of any part of the work are appropriately investigated and resolved. The study was conducted in accordance with the Declaration of Helsinki (as revised in 2013). The study was approved and monitored by the Ethics Committee of Changhai Hospital (No. CHEC2020-035). Because of the retrospective nature of the study, the requirement for informed consent was waived.

Open Access Statement: This is an Open Access article distributed in accordance with the Creative Commons Attribution-NonCommercial-NoDerivs 4.0 International License (CC BY-NC-ND 4.0), which permits the noncommercial replication and distribution of the article with the strict proviso that no changes or edits are made and the original work is properly cited (including links to both the formal publication through the relevant DOI and the license). See: https://creativecommons.org/licenses/by-nc-nd/4.0/.

\section{References}

1. Siegel RL, Miller KD, Jemal A. Cancer statistics, 2020. CA Cancer J Clin 2020;70:7-30.

2. Wieszczy P, Kaminski MF, Franczyk R, et al. Colorectal Cancer Incidence and Mortality After Removal of Adenomas During Screening Colonoscopies. Gastroenterology 2020;158:875-883.e5.

3. Weitz J, Koch M, Debus J, et al. Colorectal cancer. Lancet 2005;365:153-65.

4. Koyama M, Murata A, Sakamoto Y, et al. Risk Factors for Anastomotic Leakage After Intersphincteric Resection Without a Protective Defunctioning Stoma for Lower Rectal Cancer. Ann Surg Oncol 2016;23 Suppl 2:S249-56.

5. Rencuzogullari A, Benlice C, Valente $M$, et al. Predictors of Anastomotic Leak in Elderly Patients After Colectomy: Nomogram-Based Assessment from the American College of Surgeons National Surgical Quality Program Procedure-Targeted Cohort. Dis Colon Rectum 2017;60:527-36.

6. Yao HH, Shao F, Huang Q, et al. Nomogram to predict anastomotic leakage after laparoscopic anterior resection with intracorporeal rectal transection and double-stapling technique anastomosis for rectal cancer. Hepatogastroenterology 2014;61:1257-61.

7. Yun JA, Cho YB, Park YA, et al. Clinical manifestations and risk factors of anastomotic leakage after low anterior resection for rectal cancer. ANZ J Surg 2017;87:908-14.

8. Tan WS, Tang CL, Shi L, et al. Meta-analysis of defunctioning stomas in low anterior resection for rectal cancer. Br J Surg 2009;96:462-72.

9. Park JS, Huh JW, Park YA, et al. Risk Factors of Anastomotic Leakage and Long-Term Survival After Colorectal Surgery. Medicine (Baltimore) 2016;95:e2890.

10. Kream J, Ludwig KA, Ridolfi TJ, et al. Achieving low anastomotic leak rates utilizing clinical perfusion assessment. Surgery 2016;160:960-7.

11. Hoshino N, Hida K, Sakai Y, et al. Nomogram for predicting anastomotic leakage after low anterior resection for rectal cancer. Int J Colorectal Dis 2018;33:411-8.

12. Zheng H, Wu Z, Wu Y, et al. Laparoscopic surgery may decrease the risk of clinical anastomotic leakage and a nomogram to predict anastomotic leakage after anterior resection for rectal cancer. Int J Colorectal Dis 2019;34:319-28.

13. Zhang W, Lou Z, Liu Q, et al. Multicenter analysis of risk factors for anastomotic leakage after middle and low rectal cancer resection without diverting stoma: a retrospective study of 319 consecutive patients. Int J Colorectal Dis 2017;32:1431-7.

14. Mogensen UB, Ishwaran H, Gerds TA. Evaluating Random Forests for Survival Analysis using Prediction Error Curves. J Stat Softw 2012;50:1-23.

15. Rahbari NN, Weitz J, Hohenberger W, et al. Definition and grading of anastomotic leakage following anterior resection of the rectum: a proposal by the International Study Group of Rectal Cancer. Surgery 2010;147:339-51.

16. Austin PC, Jembere N, Chiu M. Propensity score matching and complex surveys. Stat Methods Med Res 2018;27:1240-57.

17. Carter JV, Pan J, Rai SN, et al. ROC-ing along: Evaluation and interpretation of receiver operating characteristic curves. Surgery 2016;159:1638-45.

18. Shiomi A, Ito M, Maeda K, et al. Effects of a diverting stoma on symptomatic anastomotic leakage after low anterior resection for rectal cancer: a propensity score matching analysis of 1,014 consecutive patients. J Am Coll Surg 2015;220:186-94.

19. Xiao C, Zhou M, Yang X, et al. Novel nomogram with microvascular density in the surgical margins can accurately predict the risk for anastomotic leakage after anterior resection for rectal cancer. J Surg Oncol 2019;120:1412-9.

20. Klose J, Tarantino I, von Fournier A, et al. A Nomogram 
to Predict Anastomotic Leakage in Open Rectal SurgeryHope or Hype? J Gastrointest Surg 2018;22:1619-30.

21. Cai Z, Xu D, Zhang Q, et al. Classification of lung cancer using ensemble-based feature selection and machine learning methods. Mol Biosyst 2015;11:791-800.

22. Kursa MB. Robustness of Random Forest-based gene selection methods. BMC Bioinformatics 2014;15:8.

23. Wang Y, Xia ST, Tang Q, et al. A Novel Consistent Random Forest Framework: Bernoulli Random Forests. IEEE Trans Neural Netw Learn Syst 2018;29:3510-23.

24. Poldrack RA, Huckins G, Varoquaux G. Establishment of Best Practices for Evidence for Prediction: A Review. JAMA Psychiatry 2020;77:534-40.

25. Liu XH, $\mathrm{Wu} \mathrm{XR}$, Zhou $\mathrm{C}$, et al. Conversion is a risk factor for postoperative anastomotic leak in rectal cancer patients - A retrospective cohort study. Int J Surg 2018;53:298-303.

26. Ma T, Zhong Q, Cao W, et al. Clinical Anastomotic Leakage After Rectal Cancer Resection Can Be Predicted by Pelvic Anatomic Features on Preoperative MRI Scans: A Secondary Analysis of a Randomized Controlled Trial. Dis Colon Rectum 2019;62:1326-35.

27. Yang SU, Park EJ, Baik SH, et al. Modified Colon Leakage Score to Predict Anastomotic Leakage in Patients Who

Cite this article as: Wen R, Zheng K, Zhang Q, Zhou L, Liu Q, Yu G, Gao X, Hao L, Lou Z, Zhang W. Machine learningbased random forest predicts anastomotic leakage after anterior resection for rectal cancer. J Gastrointest Oncol 2021;12(3):921932. doi: 10.21037/jgo-20-436
Underwent Left-Sided Colorectal Surgery. J Clin Med 2019;8:1450.

28. Shinji S, Ueda Y, Yamada T, et al. Male sex and history of ischemic heart disease are major risk factors for anastomotic leakage after laparoscopic anterior resection in patients with rectal cancer. BMC Gastroenterol 2018;18:117.

29. Wang XT, Li L, Kong FB, et al. Surgical-related risk factors associated with anastomotic leakage after resection for rectal cancer: a meta-analysis. Jpn J Clin Oncol 2020;50:20-8.

30. Curtis NJ, Foster JD, Miskovic D, et al. Association of Surgical Skill Assessment With Clinical Outcomes in Cancer Surgery. JAMA Surg 2020;155:590-8.

31. Akiyoshi T, Kuroyanagi H, Ueno M, et al. Learning curve for standardized laparoscopic surgery for colorectal cancer under supervision: a single-center experience. Surg Endosc 2011;25:1409-14.

32. Barrie J, Jayne DG, Wright J, et al. Attaining surgical competency and its implications in surgical clinical trial design: a systematic review of the learning curve in laparoscopic and robot-assisted laparoscopic colorectal cancer surgery. Ann Surg Oncol 2014;21:829-40. 
Supplementary

Table S1 Qualification of surgeons

\begin{tabular}{lccc}
\hline Surgeon & Years of training & Year of doing colorectal surgery independently & Year of doing laparoscopic surgery independently \\
\hline 1 & $>10$ & 2005 & - \\
2 & $>10$ & 2007 & 2009 \\
3 & $>10$ & 1989 & 2017 \\
4 & $>10$ & 2000 & 2008 \\
5 & $>10$ & 2011 & 2014 \\
6 & $>10$ & 2013 & 2013 \\
7 & $>10$ & 2006 & 2008 \\
8 & $>10$ & 2014 & 2015 \\
\hline
\end{tabular}




\begin{tabular}{|c|c|c|c|}
\hline Variables & Patients without AL ( $=791$ ) & Patients with AL (n=45) & $P$ value \\
\hline $\mathrm{BMI}, \mathrm{kg} / \mathrm{m}^{2}$ & $23.52 \pm 2.99$ & $23.95 \pm 3.02$ & 0.35 \\
\hline Sex & & & 0.14 \\
\hline Female & $267(96.4)$ & $10(3.6)$ & \\
\hline Male & $524(93.7)$ & $35(6.3)$ & \\
\hline Age & $60.49 \pm 10.77$ & $59.78 \pm 10.17$ & 0.67 \\
\hline Blood transfusion, $\mathrm{mL}$ & $20.23 \pm 158.78$ & $35.56 \pm 166.73$ & 0.53 \\
\hline Surgeon volume, /year & & & 0.04 \\
\hline$\geq 100$ & 749 (95.1) & $39(4.9)$ & \\
\hline$<100$ & $42(87.5)$ & $6(12.5)$ & \\
\hline Blood loss, $\mathrm{mL}$ & $166.22 \pm 149.70$ & $170.00 \pm 151.66$ & 0.87 \\
\hline Surgical approach & & & 0.93 \\
\hline Open & $611(94.6)$ & $35(5.4)$ & \\
\hline Laparoscopic & $180(94.7)$ & $10(5.3)$ & \\
\hline Stoma & & & 0.03 \\
\hline No & $250(97.3)$ & $7(2.7)$ & \\
\hline Yes & $541(93.4)$ & $38(6.6)$ & \\
\hline nCRT & & & 0.08 \\
\hline No & $586(95.4)$ & $28(4.6)$ & \\
\hline Yes & $205(92.3)$ & $17(7.7)$ & \\
\hline Distance, $\mathrm{cm}$ & $6.60 \pm 3.28$ & $5.36 \pm 3.09$ & 0.01 \\
\hline Stenosis or obstruction & & & 0.52 \\
\hline No & $527(95.0)$ & $28(5.0)$ & \\
\hline Yes & $264(94.0)$ & $17(6.0)$ & \\
\hline Pathological types & & & 0.26 \\
\hline Well differentiated & $20(95.2)$ & $1(4.8)$ & \\
\hline Moderately differentiated & 647 (94.6) & $37(5.4)$ & \\
\hline Poorly differentiated & 37 (88.1) & $5(11.9)$ & \\
\hline Mucinous & $80(97.6)$ & $2(2.4)$ & \\
\hline Signet ring cell & $7(100.0)$ & $0(0.0)$ & \\
\hline pT stage & & & 0.11 \\
\hline 1,2 & $275(92.9)$ & $21(7.1)$ & \\
\hline 3,4 & $516(95.6)$ & $24(4.4)$ & \\
\hline $\mathrm{pN}$ stage & & & 0.64 \\
\hline 0 & 468 (94.9) & $25(5.1)$ & \\
\hline 1,2 & $323(94.2)$ & $20(5.8)$ & \\
\hline pALB, g/L & & & 0.62 \\
\hline$\geq 35$ & $771(94.5)$ & $45(5.5)$ & \\
\hline$<35$ & $20(100.0)$ & $0(0.0)$ & \\
\hline pHGB, g/L & & & 0.04 \\
\hline$\geq 90$ & 779 (94.9) & $42(5.1)$ & \\
\hline$<90$ & $12(80.0)$ & $3(20.0)$ & \\
\hline Diabetes & & & 0.007 \\
\hline No & $662(95.7)$ & $30(4.3)$ & \\
\hline Yes & $129(89.6)$ & $15(10.4)$ & \\
\hline ASA score & & & 0.31 \\
\hline$\leq 2$ & 711 (94.9) & $38(5.1)$ & \\
\hline$\geq 3$ & $80(92.0)$ & $7(8.0)$ & \\
\hline CA199, U/mL & & & 0.21 \\
\hline$\leq 37$ & 712 (94.9) & $38(5.1)$ & \\
\hline$>37$ & 79 (91.9) & $7(8.1)$ & \\
\hline CEA, ng/mL & & & 0.14 \\
\hline$\leq 5$ & $525(93.8)$ & $35(6.3)$ & \\
\hline$>5$ & 266 (94.6) & $10(3.6)$ & \\
\hline
\end{tabular}

Data were presented as $n(\%)$ or mean \pm standard deviation. AL, anastomotic leakage; BMI, body mass index; $\mathrm{nCRT}$, neoadjuvant chemoradiotherapy; pALB, preoperative albumin; pHGB, preoperative hemoglobin; ASA, American Society of Anesthesiologists; CEA, carcinoembryonic antigen; CA199, carbohydrate antigen 199. 
Table S3 Univariate analysis of variables related to stoma $(\mathrm{n}=5,220)$

\begin{tabular}{|c|c|c|c|}
\hline Variables & Patients without stoma $(n=1,766)$ & Patients with stoma $(n=3,454)$ & $P$ value \\
\hline $\mathrm{BMI}, \mathrm{kg} / \mathrm{m}^{2}$ & $23.02 \pm 2.98$ & $23.52 \pm 3.16$ & $<0.001$ \\
\hline Sex & & & $<0.001$ \\
\hline Female & $711(39.0)$ & $1,113(4.4)$ & \\
\hline Male & $1,055(31.1)$ & $2,341(68.9)$ & \\
\hline Age & $59.88 \pm 11.43$ & $59.48 \pm 11.31$ & 0.23 \\
\hline Blood transfusion, $\mathrm{mL}$ & $22.37 \pm 133.90$ & $33.32 \pm 181.64$ & 0.01 \\
\hline Surgeon volume, /year & & & 0.59 \\
\hline$\geq 100$ & $1,551(33.7)$ & $3,052(66.3)$ & \\
\hline$<100$ & $215(34.8)$ & $402(65.2)$ & \\
\hline Blood loss, mL & $183.60 \pm 108.41$ & $205.38 \pm 165.66$ & $<0.001$ \\
\hline Surgical approach & & & 0.048 \\
\hline Open & $1,437(33.2)$ & $2,887(66.8)$ & \\
\hline Laparoscopic & $329(36.7)$ & $567(63.3)$ & \\
\hline nCRT & & & $<0.001$ \\
\hline No & $1,373(35.4)$ & $2,504(64.6)$ & \\
\hline Yes & $393(29.3)$ & $950(70.7)$ & \\
\hline Distance, $\mathrm{cm}$ & $9.42 \pm 2.92$ & $5.76 \pm 2.52$ & $<0.001$ \\
\hline Stenosis or obstruction & & & 0.06 \\
\hline No & $1,207(34.9)$ & $2,256(65.1)$ & \\
\hline Yes & $659(37.5)$ & $1,098(62.5)$ & \\
\hline Pathological types & & & 0.35 \\
\hline Well differentiated & $44(32.1)$ & $93(67.9)$ & \\
\hline Moderately differentiated & $1,440(33.9)$ & $2,810(66.1)$ & \\
\hline Poorly differentiated & $86(35.4)$ & $157(64.6)$ & \\
\hline Mucinous & $191(34.1)$ & $369(65.9)$ & \\
\hline Signet ring cell & $5(16.7)$ & 25 (83.3) & \\
\hline pT stage & & & 0.30 \\
\hline 1,2 & $698(36.7)$ & $1,202(63.3)$ & \\
\hline 3,4 & $1,268(38.2)$ & $2,052(61.8)$ & \\
\hline $\mathrm{pN}$ stage & & & 0.84 \\
\hline 0 & $1,083(35.9)$ & $1,936(64.1)$ & \\
\hline 1,2 & $783(35.6)$ & $1,418(64.4)$ & \\
\hline pALB, g/L & & & 0.04 \\
\hline$\geq 35$ & $1,674(33.5)$ & $3,318(66.5)$ & \\
\hline$<35$ & $92(40.4)$ & $136(59.6)$ & \\
\hline $\mathrm{pHGB}, \mathrm{g} / \mathrm{L}$ & & & 0.16 \\
\hline$\geq 90$ & $1,721(33.7)$ & $3,387(66.3)$ & \\
\hline$<90$ & $45(40.2)$ & $67(59.8)$ & \\
\hline Diabetes & & & 0.10 \\
\hline No & $1,561(34.3)$ & $2,996(65.7)$ & \\
\hline Yes & $205(30.9)$ & $458(69.1)$ & \\
\hline ASA score & & & 0.73 \\
\hline$\leq 2$ & $1,610(33.9)$ & $3,139(66.1)$ & \\
\hline$\geq 3$ & $156(33.1)$ & $315(66.9)$ & \\
\hline CA199, U/mL & & & 0.33 \\
\hline$\leq 37$ & $1,880(41.2)$ & $2,679(58.8)$ & \\
\hline$>37$ & $286(43.3)$ & $375(56.7)$ & \\
\hline $\mathrm{CEA}, \mathrm{ng} / \mathrm{mL}$ & & & 0.07 \\
\hline$\leq 5$ & $1,141(33.0)$ & $2,319(67.0)$ & \\
\hline$>5$ & 625 (35.5) & $1,135(64.5)$ & \\
\hline
\end{tabular}

Data were presented as $n(\%)$ or mean \pm standard deviation. $A L$, anastomotic leakage; BMI, body mass index; $n C R T$, neoadjuvant chemoradiotherapy; pALB, preoperative albumin; pHGB, preoperative hemoglobin; ASA, American Society of Anesthesiologists; CEA, carcinoembryonic antigen; CA199, carbohydrate antigen 199. 
Table S4 Relationship between stoma and reoperation ( $\mathrm{n}=326)$

\begin{tabular}{lccc}
\hline Stoma & Non-reoperation & Reoperation & P value \\
\hline No & $46(63.0 \%)$ & $27(37.0 \%)$ & \\
Yes & $251(99.2 \%)$ & $2(0.8 \%)$ & $<0.001$ \\
\hline
\end{tabular}

Table S5 Relationship between KRAS and AL (n=3,806)

\begin{tabular}{lccc}
\hline KRAS & Patient without AL & Patient with AL & P value \\
\hline Wild & $2,002(93.8 \%)$ & $132(6.2 \%)$ & \\
Mutant & $1,573(94.1 \%)$ & $99(5.9 \%)$ & 0.784 \\
\hline
\end{tabular}

$\mathrm{AL}$, anastomotic leakage. 